Check for updates

Cite this: RSC Adv., 2019, 9, 13026

\title{
NIR laser-responsive liquid metal-loaded polymeric hydrogels for controlled release of doxorubicin $\dagger$
}

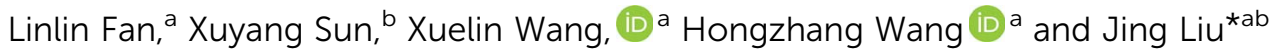

Liquid metals (LMs) have recently emerged as a new class of promising multifunctional materials with attractive properties. They have excellent photothermal conversion efficiency, generating heat under near-infrared (NIR) laser irradiation. This work reports encapsulating LM droplets into poly(NIPAm-COMBA) hydrogels (PNM) to achieve nanodispersed liquid metals in bulk polymeric hydrogels for NIR laserresponsive materials. $L M$ droplets $(\sim 530 \mathrm{~nm})$ are produced by dispersing an alloy of gallium and indium (EGaln) into glycerol. The LM-loaded PNM hydrogels (PNM/LM) exhibited excellent thermal-/NIR laserresponsive ability. In a water bath, the weight of the PNM/LM can decrease $92 \%$ at $50{ }^{\circ} \mathrm{C}$. And the volume of PNM/LM can decrease $62 \%$ under NIR laser irradiation for $12 \mathrm{~min}$. Because of its thermal-/NIR laser-responsive ability and porous three-dimensional (3D) networks, PNM/LM is very suitable for use as a drug carrier. We also prepared doxorubicin (DOX)-loaded PNM/LM hydrogels (PNM/LM/DOX) and demonstrated that the PNM/LM/DOX hydrogel can generate heat and raise its temperature under NIR laser irradiation. When the temperature becomes higher than the lower critical solution temperature (LCST), such a hydrogel would shrink immediately and extrude the DOX encapsulated in its networks simultaneously, then complete the controlled release of the pre-loaded drug. Further, an in vitro cytotoxicity test indicated the biocompatibility and feasibility as a chemophotothermal synergistic therapeutic of the present hydrogel. This NIR laser-responsive hydrogel fully exhibits its superiority as a drug carrier which promises great potential in future targeted controlled drug release.
\end{abstract}

Received 25th March 2019
Accepted 16th April 2019
DOI: 10.1039/c9ra02286d
rsc.li/rsc-advances undergo a phase transition to hydrophobic aggregation above its LCST due to the presence of hydrophilic amide groups and hydrophobic isopropyl groups. ${ }^{7,8}$ PNIPAm-based hydrogels owns a LCST of about $32{ }^{\circ} \mathrm{C},,^{\mathbf{9} 10}$ which is lower than the human body temperature $\left(37^{\circ} \mathrm{C}\right)$. So, PNIPAm and its hydrogels have been studied for the "on-off" drug release utilizing this thermalresponsive property. ${ }^{\mathbf{1 0 , 1 1}}$ However, there are also some disadvantages. The inner of these conventional thermal-responsive materials such as PNIPAm and its hydrogels is hard to respond to the outside temperature changes due to their poor thermal conductivity. In order to improve its thermal sensitivity as well as promote its respond speed, some nanoparticles with photothermal conversion capability under near-infrared (NIR) laser irradiation, such as gold nanoparticles ${ }^{\mathbf{1 2 , 1 3}}$ and reduced graphene oxide, ${ }^{\mathbf{1 4 , 1 5}}$ are incorporated into hydrogels to endow them multi-functions. Additionally, liquid metals (LMs) also have this photothermal conversion capability. ${ }^{16} \mathrm{LM}$ is a special family of materials that have both metallic and fluidic properties simultaneously. ${ }^{17,18}$ Typical materials in the area such as gallium, gallium-indium eutectic alloys (EGaIn) and galliumindium-tin alloys (Galinstan), which have low melting points, have been widely used as printed circuits, soft electronics, and other biomaterials. ${ }^{19-24}$ EGaIn preserves the essential characteristics of LMs, such as excellent combination of thermalconductivity and transformability, while presenting better
${ }^{a}$ Department of Biomedical Engineering, School of Medicine, Tsinghua University, Beijing 100084, China. E-mail: jliubme@tsinghua.edu.cn

${ }^{b}$ Technical Institute of Physics and Chemistry, Chinese Academy of Sciences, Beijing 100190, China

$\dagger$ Electronic supplementary information (ESI) available. See DOI: 10.1039/c9ra02286d 
biocompatibility. ${ }^{18}$ Therefore, the NIR laser-responsive hydrogels can be produced by introducing LMs into thermalresponsive materials. These hydrogels can respond to temperature changes caused by NIR laser irradiation, aiming to inducing changes in the structure of hydrogels by photothermal effect and achieving targeted controlled release of drugs. In this study, we synthesized a poly(NIPAm-co-MBA) hydrogel (PNM) contained with LM droplets. The thermal-responsive PNM has a porous 3D network structure, which can lock LM droplets tightly. LM droplets can promote the response of hydrogels to NIR laser irradiation. Therefore, the LM-loaded PNM hydrogels (PNM/LM) exhibited excellent thermal-/NIR laser-responsive ability. Because of its thermal-/NIR laser-responsive ability and porous 3D networks, the PNM/LM is very suitable for using as drug carriers. We also prepared doxorubicin (DOX)-loaded PNM/LM hydrogels (PNM/LM/DOX). As shown in Scheme 1, the PNM/LM/DOX can absorb NIR light and transform to thermal energy under irradiation. NIR laser irradiation causes the temperature to rise above LCST, leading to changes of hydrogel shape and size. The hydrogel shrinks and extrudes the aqueous solution and pre-loaded DOX from hydrogels simultaneously. In this way, the hydrogel could complete the controlled release of DOX constantly and smoothly, reducing side effects of unnecessary drug leaks in normal tissues. So, the $\mathrm{PNM} / \mathrm{LM}$ is a "smart" polymeric hydrogel for anticancer drug delivery and chemophotothermal synergistic therapy. And a series of experimental test results demonstrate that this NIR laser-responsive hydrogel PNM/LM is a promising carrier for drug delivery system.

\section{Experimental}

\section{Materials}

A gallium (Ga) and indium (In) were purchased from local company (China). $N$-Isopropylacrylamide (NIPAm) and glycerol were obtained from Shanghai Macklin Biochemical Co. Ltd. (Shanghai, China). $N, N^{\prime}$-Methylenebis(acrylamide) (MBA) was purchased from Sigma-Aldrich (WUXI) Life Science \& Technology Co. Ltd. (Wuxi, China). Ammonium persulfate (APS) was obtained from Aladdin Industrial Corporation (Shanghai, China). Doxorubicin hydrochloride $(\mathrm{DOX} \cdot \mathrm{HCl})$ was purchased from Shanghai D\&B Biological Science and Technology Co. Ltd. (Shanghai, China). All the chemicals were pure and used

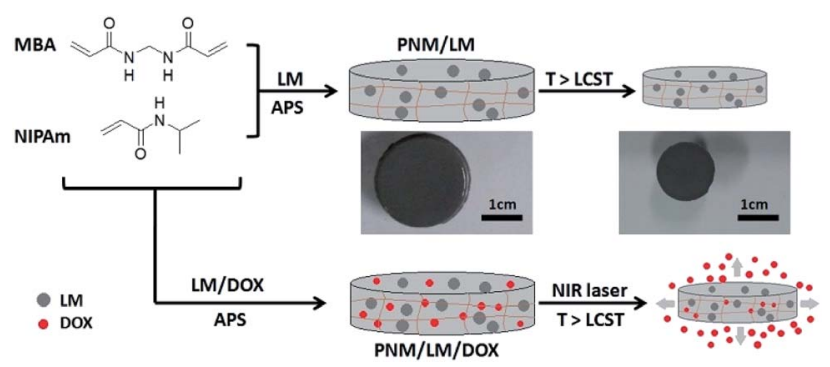

Scheme 1 Schematic illustration of the formation of NIR laserresponsive hydrogels (PNM/LM and PNM/LM/DOX) and the release of DOX. directly without further purifying. Dulbecco's Modification of Eagle's Medium (DMEM) was purchased from Wisent (China). Fetal bovine serum (FBS), penicillin/streptomycin and phosphate buffered saline (PBS, $\mathrm{pH}$ 7.4) were obtained from Thermo Fisher Scientific Corporation (China). Cell counting kit-8 (CCK8) was purchased from Dojindo Laboratories (China). Acridine orange (AO) and ethidium bromide (EB) solution were obtained from Solarbio (China). 4T1 mouse breast cancer cells were supplied by American Type Culture Collection (ATCC).

\section{Preparation of liquid metal}

Liquid metal was composed of gallium and indium. The EGaIn alloy was prepared with a weight ratio of $75.5 \%(\mathrm{Ga})$ and $24.5 \%$ (In). The metals were mixed at $100{ }^{\circ} \mathrm{C}$ with a continuous stirrer till fused completely.

\section{Preparation of liquid metal droplets}

Bulk EGaIn was added into glycerol, it was dispersed into droplets under ultrasonication in ice-water bath. The sonication time and glycerol dosage were tuned to adjust the size of LM droplets.

Synthesis of poly(NIPAm-co-MBA) polymeric hydrogels with or without LM droplets and DOX

Thermal-responsive polymeric hydrogels were synthesized by copolymerization of NIPAm and MBA with APS as the initiator.

To prepare blank hydrogels poly(NIPAm-co-MBA) (PNM), NIPAm (1.0 g) and MBA (0.1 g) were dissolved in deionized water with magnetic stirrer. After the monomer dissolved in the solution, APS aqueous solution was added into the mixture solution to initiate polymerization. The polymerization was completed at $60{ }^{\circ} \mathrm{C}$ for $10 \mathrm{~min}$.

To prepare LM-loaded poly(NIPAm-co-MBA) hydrogels (PNM/ LM), NIPAm (1.0 g), MBA (0.1 g) and LM droplets (EGaIn $0.5 \mathrm{~g}$ ) were added in deionized water with magnetic stirrer. After that, APS aqueous solution was added into the mixture solution to initiate polymerization. The polymerization was completed at room temperature $\left(25^{\circ} \mathrm{C}\right)$ for $10 \mathrm{~min}$.

To prepare LM-and DOX-loaded poly(NIPAm-co-MBA) hydrogels (PNM/LM/DOX), DOX· $\mathrm{HCl}(20 \mathrm{mg})$ was dissolved in DMSO $(50 \mu \mathrm{l})$ and then transferred to deionized water $(400 \mu \mathrm{l})$, NIPAm (1.0 g), MBA (0.1 g), LM droplets (EGaIn $0.5 \mathrm{~g}$ ) and $\mathrm{DOX} \cdot \mathrm{HCl}$ aqueous solution were added in deionized water with magnetic stirrer. After that, APS aqueous solution was added into the mixture solution to initiate polymerization. The polymerization was completed at room temperature $\left(25{ }^{\circ} \mathrm{C}\right)$ for 20 min. DOX-loaded poly(NIPAm-co-MBA) hydrogels (PNM/ DOX) was prepared following the same procedure.

All hydrogels were immersed into deionized water to remove low molecular compound.

\section{Characterization of liquid metal droplets}

LM droplets size and surface morphology were observed by scanning electron microscopy (SEM, Quanta 200, Holland) and transmission electron microscopy (TEM, H-7650B, Japan). The 
hydrodynamic size was measured with dynamic light scattering (DLS, DynaPro NanoStar, USA).

\section{Characterization of polymeric hydrogels}

To test selling ratio, the dried hydrogels were immersed into deionized water of different temperatures for $24 \mathrm{~h}$. They were measured swelling weight after wiping off the excessive water from hydrogels surface. The swelling ratio $(S)$ was calculated according to the following equation $S=\left(W_{\mathrm{s}}-W_{\mathrm{d}}\right) / W_{\mathrm{d}} \times 100 \%$, where $W_{\mathrm{s}}$ and $W_{\mathrm{d}}$ are the weight of swelling and dried hydrogels, respectively.

The surface morphology of the synthesized PNM and PNM/ LM hydrogels were observed by SEM. Lyophilizing hydrogel samples were processed by freeze dryer before testing. The rheology measurement of polymeric hydrogels was conducted with rheometer (MCR301, Austria). Cylindrical hydrogels (25 $\mathrm{mm}$ diameter) were put on the bottom plate. The storage modulus $\left(G^{\prime}\right)$ and loss modulus $\left(G^{\prime \prime}\right)$ of polymeric hydrogels were monitored over a shear strain ranging from $0.01 \%$ to $10 \%$ with a fixed frequency $(1 \mathrm{~Hz})$ at $25{ }^{\circ} \mathrm{C}$. Then, the $G^{\prime}$ and $G^{\prime \prime}$ of hydrogels were monitored over a frequency ranging from 0.1 to $10 \mathrm{~Hz}$ at a shear strain of $0.74 \%$ at $37^{\circ} \mathrm{C}$.

To test the NIR laser-responsive behavior of hydrogels, blank hydrogels and LM-loaded hydrogels were exposed to a NIR laser at different power density $\left(0.5,0.8,1.0,1.2\right.$ and $\left.1.5 \mathrm{~W} \mathrm{~cm}^{-2}\right)$. The ultraviolet-visible-NIR absorption spectrum of bulk LM, LM droplets and PNM/LM from 500 to $1000 \mathrm{~nm}$ was tested to verify their absorption peak at around $800 \mathrm{~nm}$ in the NIR region. ${ }^{16} \mathrm{~A}$ wavelength of $808 \mathrm{~nm}$ was used for the NIR laser-responsive assay. The irradiation area of the laser was a circle spot. Temperature change was measured and recorded by a thermocouple thermometer every $10 \mathrm{~s}$ automatically. PBS was used as the control. Volume change was measured by vernier caliper. The dynamic volume change ratios $\left(V_{t} / V_{0}\right)$ were calculated by the equation $V_{t} / V_{0}=\left(d_{t} / d_{0}\right)^{3}$, where $d_{t}$ is the diameter of hydrogels at time $t$, and $d_{0}$ is the diameter of hydrogels at beginning.

All tests were conducted in triplicates.

\section{In vitro drug release}

DOX-loaded hydrogel PNM/DOX and PNM/LM/DOX were immersed into PBS ( $\mathrm{pH}$ 7.4) at different temperature from 20 to $70{ }^{\circ} \mathrm{C}$. The released drug in PBS was collected and measured by absorbance at $480 \mathrm{~nm}\left(\mathrm{OD}_{480} \mathrm{~nm}\right)$ through microplate assay (Envision, UK). To test the cumulative DOX release in PBS at $37{ }^{\circ} \mathrm{C}, \mathrm{PNM} / \mathrm{DOX}$ and PNM/LM/DOX hydrogels were immersed into PBS for $24 \mathrm{~h}$. At certain time intervals, the DOX release medium was collected and an equal volume of fresh PBS was added.

To test NIR laser-responsive drug release behavior, the PNM/ LM/DOX hydrogel was immersed into PBS and exposed to an NIR laser irradiation for $12 \mathrm{~min}\left(808 \mathrm{~nm}, 1.0 \mathrm{~W} \mathrm{~cm}^{-2}\right)$ at the time points of $0,4,8,12,16$ and $20 \mathrm{~h}$, respectively. The released drug in PBS was collected and measured through microplate assay.

All tests were conducted in triplicates.

\section{In vitro cytotoxicity assay}

Cell viabilities were determined using cell counting kit-8 (CCK8) assay with $4 \mathrm{~T} 1$ cancer cells. Cells were cultured in Dulbecco's Modification of Eagle's Medium (DMEM) containing 10\% fetal bovine serum (FBS) and $1 \%$ penicillin/streptomycin at $37{ }^{\circ} \mathrm{C}$ in a humidified $5 \% \mathrm{CO}_{2}$ atmosphere. Cells were plated at a density of 5000 cells per well in 96-well plates and cultured in an incubator for $24 \mathrm{~h}$. Then, hydrogels to be tested were placed in wells containing medium with plastic tray. To research the cytotoxicity and tumor therapy synergistic effect of hydrogels, cells were treated with blank hydrogel (PNM), LM-loaded hydrogel (PNM/LM) and LM and DOX-loaded hydrogel (PNM/ LM/DOX), with or without NIR laser irradiation $(808 \mathrm{~nm}$, $1.0 \mathrm{~W} \mathrm{~cm}^{-2}$ ) for $12 \mathrm{~min}$. Cells incubated with PNM/DOX or $\mathrm{PNM} / \mathrm{LM} / \mathrm{DOX}$ hydrogels exposed to NIR laser irradiation (808 $\mathrm{nm}, 1.0 \mathrm{~W} \mathrm{~cm}^{-2}$ ) for 0, 1, 2, 5, 8 and $12 \mathrm{~min}$. Cells grown in absence of hydrogels and exposed at NIR laser irradiation (808 $\mathrm{nm}, 1.0 \mathrm{~W} \mathrm{~cm}^{-2}$ ) was used as the control. Then the medium was removed and $100 \mu \mathrm{l}$ of CCK- 8 medium solution was added to each well, cells were incubated for another $2 \mathrm{~h}$ at $37^{\circ} \mathrm{C}$. The absorbance at $450 \mathrm{~nm}\left(\mathrm{OD}_{450 \mathrm{~nm}}\right)$ was determined by microplate reader.

Cell viability was observed using a laser scanning confocal microscope (Zeiss LSM710, China). Cells were treated with previous procedures. Then cells were treated with AO-EB solution (staining live cell green, staining dead cells red). Finally, each well was washed with PBS three times.

\section{Statistical analysis}

Results were analyzed by one-way ANOVA tests and differences were considered significant at $P \leq 0.05$.

\section{Results and discussions}

\section{Preparation and characterization of liquid metal droplets}

LM droplets are not chemically stable under aqueous conditions, because gallium tends to generate $\mathrm{GaOOH}$ or $\mathrm{Ca}_{2} \mathrm{O}_{3}$ immediately when exposed to water and oxygen. ${ }^{18,25}$ Gallium can form a film of $\mathrm{Ga}_{2} \mathrm{O}_{3}$ on its surface to prevent further chemical reactions in aqueous solution and then further produce $\mathrm{GaOOH}$ in alkaline solution. ${ }^{26}$ And $\mathrm{LM}$ droplets tend to agglomerate to large one in water. So, it is difficult to keep in a small size. In this study, LM droplets were prepared by dispersing bulk EGaIn into glycerol under ultrasonication in ice-water bath. These droplets could be well dispersed and kept stable due to the high viscosity of glycerol. LM droplets were characterized by SEM (Fig. 1A) and TEM (Fig. 1B) image. They revealed the uniform spherical morphology of LM droplets. The hydrodynamic diameter of the LM droplets was determined by dynamic light scattering (DLS) as $\sim 530 \mathrm{~nm}$ (Fig. 1C), and this size did not change for at least 10 days at room temperature.

\section{Thermal effect of polymeric hydrogels}

The PNM was synthesized by copolymerization of NIPAm and MBA with APS, in which MBA was used as the cross-linker and APS was adopted as the initiator. The polymeric hydrogel 

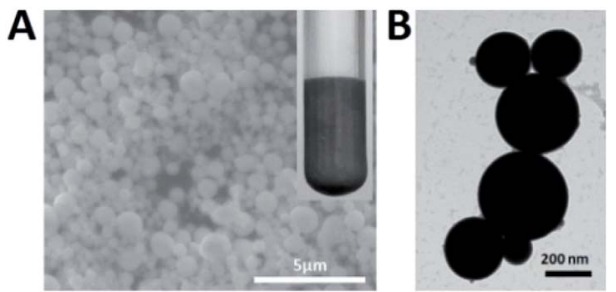

C

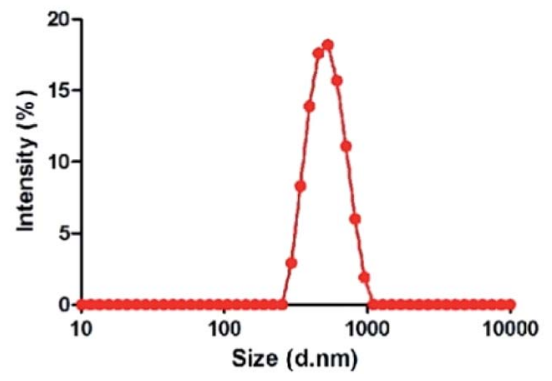

Fig. 1 Characterization of LM droplets. (A) SEM (B) TEM images of LM droplets. (C) DLS analysis of LM droplets, where size is hydrodynamic diameter.

synthesized with LM droplets could be formed at room temperature. Without LM droplets, it must be heated at $60{ }^{\circ} \mathrm{C}$. In certain extent, liquid metal acted as catalysts and it could accelerate the completion of polymerization. ${ }^{27}$

Due to its 3D network structure, the PNM/LM had good performance of water absorption and loss. As shown in Fig. 2A,
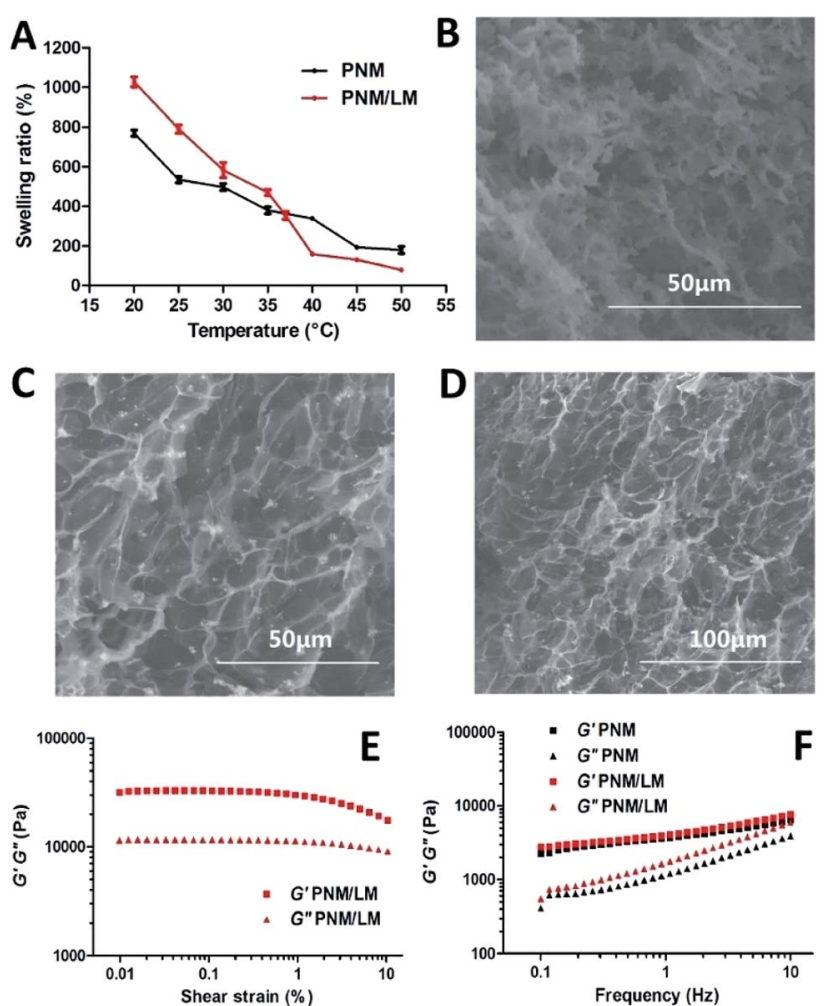

Fig. 2 Thermal-responsive test and morphologies of hydrogels. (A) Swelling ratios of PNM and PNM/LM. (B) SEM images of PNM. (C) and (D) PNM/LM. (E) Shear strain dependency of the $G^{\prime}$ and $G^{\prime \prime}$. (F) Frequency dependency of the $G^{\prime}$ and $G^{\prime \prime}$. the PNM/LM had better water absorption than PNM below $37^{\circ} \mathrm{C}$. When the temperature becomes above $37^{\circ} \mathrm{C}$, the swelling ratio of PNM/LM was lower than PNM hydrogels. It indicated that the PNM/LM can swell more when it absorbs water. At this time, the network pore in hydrogels can expand to contain more drugs. With the increase of temperature, say above $37^{\circ} \mathrm{C}$, the PNM/LM becomes shrunk and extrudes to release the drug it encapsulated before. And the higher the temperature, the more it shrinks. The weight of the PNM/LM can decrease $92 \%$ at $50{ }^{\circ} \mathrm{C}$ (Fig. 2A). Volume changes of hydrogels contained with or without LM droplets under equal water bath at different temperatures are shown in Fig. S1 and S2. $\dagger$ For the PNM/LM, it could be remain in a highly swollen state below $37{ }^{\circ} \mathrm{C}$ and a highly collapsed state above $37{ }^{\circ} \mathrm{C}$. Therefore, it is rather suitable to be applied as a carrier for drug delivery. The swelling ratio of the PNM/LM decreased dramatically between 35 and $40{ }^{\circ} \mathrm{C}$. Within this temperature range, it became above LCST $\left(\sim 32{ }^{\circ} \mathrm{C}\right)$. It confirmed that once the PNM/LM reaches human body temperature $\left(\sim 37{ }^{\circ} \mathrm{C}\right)$, it will respond immediately and shrink. As shown in Fig. 2A and S1, $\dagger$ the PNM/LM has greater volume shrinkage than PNM. It can be shrunk much smaller under the same conditions. This also confirms that the PNM/ LM has better water absorption performance and drug loading capacity than PNM as previously mentioned.

The morphologies of hydrogels contained with or without LM droplets were assessed by SEM. As shown in Fig. 2B-D, the PNM and PNM/LM all have porous structures. These pores can be used to encapsulate water and drugs. In Fig. $2 \mathrm{C}$ and $\mathrm{D}$, the bright dots are LM droplets, and they are uniform dispersion in the PNM/LM. There is no obvious LM agglomeration in PNM/ LM. As shown in Fig. $2 \mathrm{E}$ and F, the value of $G^{\prime}$ was much higher than that of their corresponding $G^{\prime \prime}$, indicating that they were viscoelastic. With the increase of frequency, the $G^{\prime \prime}$ of PNM/LM was slightly higher than that of PNM, which meant the incorporation of LM droplets could increase the viscosity of hydrogels because of the flexibility of LM. ${ }^{11}$

\section{NIR laser-responsive property of PNM/LM}

LM can generate heat under NIR laser irradiation because of the optical absorption in NIR region, and NIR laser exposure induced a transformation in LM shape. ${ }^{16}$ Therefore, the thermal-responsive hydrogel combined with LM droplets exhibit excellent NIR-laser responsive properties. To test these properties, PNM and PNM/LM were exposed to NIR laser ( $808 \mathrm{~nm}, 1.0 \mathrm{~W} \mathrm{~cm}^{-2}$ ) for $200 \mathrm{~s}$. PBS was used as control. As shown in Fig. 3A, the temperature of PNM/LM rose rapidly. For PNM, there was no significant temperature rise under the same irradiation. For testing the photothermal conversion efficiency of LM droplets in hydrogels, the PNM/LM was exposed to NIR laser $(808 \mathrm{~nm})$ at power densities from 0.5 to $1.5 \mathrm{~W} \mathrm{~cm}^{-2}$. The test results are shown in Fig. 3B, the temperature of PNM/LM increased at NIR laser irradiation power densities above $0.5 \mathrm{~W}$ $\mathrm{cm}^{-2}$. NIR laser power densities of 1.0 and $1.5 \mathrm{~W} \mathrm{~cm}^{-2}$ can increase the temperatures of hydrogels to about 57.4 and $81.6^{\circ} \mathrm{C}$ in $3 \mathrm{~min}$, respectively. With the increase of temperature, the volume of PNM/LM also changed. Under the NIR laser 

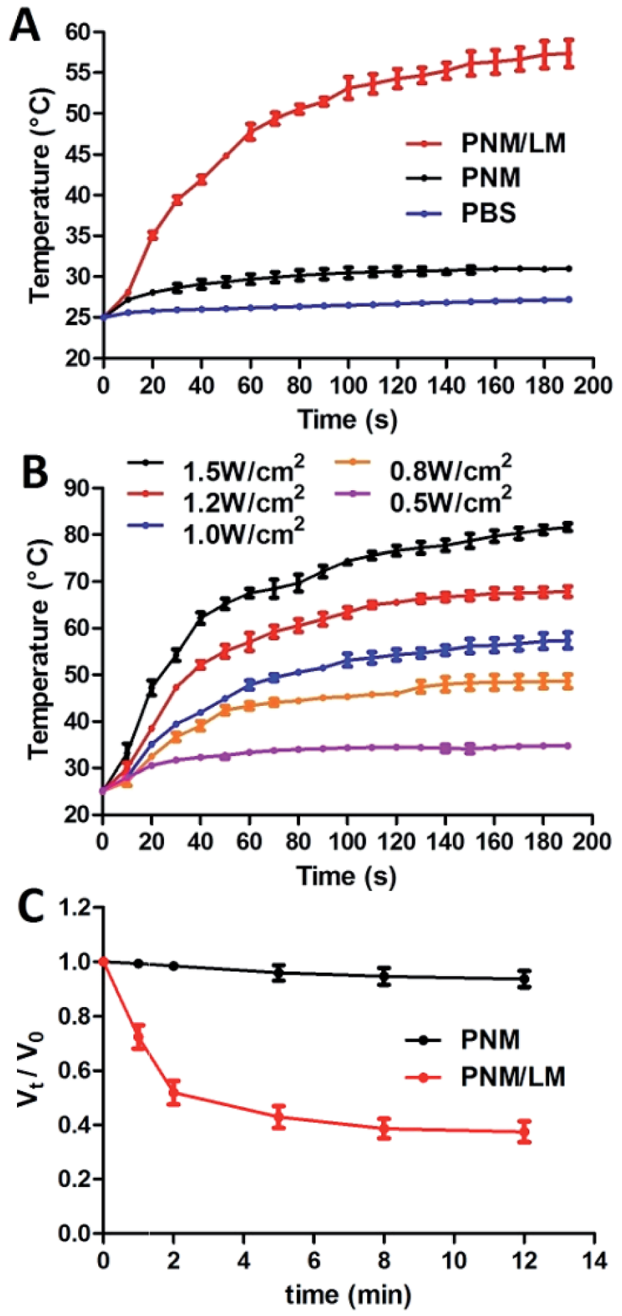

Fig. 3 NIR laser response of hydrogels. (A) The temperature change of PNM/LM, PNM and PBS exposing to NIR laser irradiation $(808 \mathrm{~nm}$, $1.0 \mathrm{~W} \mathrm{~cm}^{-2}$ ). (B) The temperature change of PNM/LM exposing to NIR laser irradiation at different power densities $(808 \mathrm{~nm}, 1.5,1.2,1.0,0.8$ and $0.5 \mathrm{~W} \mathrm{~cm}^{-2}$ ). (C) The volume change of PNM/LM and PNM exposing to NIR laser irradiation $\left(808 \mathrm{~nm}, 1.0 \mathrm{~W} \mathrm{~cm}^{-2}\right)$.

irradiation (808 $\mathrm{nm}, 1.0 \mathrm{~W} \mathrm{~cm}^{-2}$ ), the PNM/LM surface temperature increased rapidly. When the temperature increased to above the LCST $\left(\sim 32{ }^{\circ} \mathrm{C}\right)$ of PNM/LM, they also shrunk quickly, resulting in a dramatic volume decrease. As shown in Fig. 3C, the rapid shrinkage of PNM/LM occurred in the first $2 \mathrm{~min}$ of NIR laser irradiation, and then tended to balance. The volume of PNM/LM can decrease $62 \%$ under NIR laser irradiation for $12 \mathrm{~min}$ (Fig. 3C and S3†). However, PNM had no obvious volume changes. It means that the rapid NIR laser response of hydrogels is realized by adding LM droplets. LM droplets can complete the photothermal conversion successfully and effectively.

\section{Investigation of in vitro drug release}

Due to the use of cross-linker, PNM and PNM/LM all have porous structures. This structure will help them absorb water and load drugs. Hydrogels could encapsulate drugs tightly below their LCST $\left(\sim 32{ }^{\circ} \mathrm{C}\right)$. However, when the hydrogels reach the swelling equilibrium, drug leaks could occur because pores of hydrogels are bulged by aqueous solution. Once the temperature of hydrogels exceeds their LCST, these hydrogels shrink. When hydrogels shrink, their pores become small, causing extrusion of drugs and aqueous solution. In order to study the drug release effect of PNM and PNM/LM, the same dosage of DOX was carried by these hydrogels. And then PNM/ DOX and PNM/LM/DOX were put in PBS under the same conditions. As shown in Fig. 4A, PNM/DOX and PNM/LM/DOX released DOX slowly below $30{ }^{\circ} \mathrm{C}$. Free DOX remaining in networks of hydrogels was released slowly into PBS through passive diffusion. ${ }^{\mathbf{1 1}}$ Once the temperature is above LCST, hydrogels have a shrinking process, which extruded the water along with the embedded DOX in networks out of hydrogels, and achieve a rapid DOX release. It also shows that these hydrogels have a good DOX release when they are applied to human body because of body temperature. Because the PNM/ LM has better drug loading capacity and volume shrinkage performance than PNM as previously mentioned, PNM/LM/ DOX could accomplish more DOX release. In order to study DOX release of hydrogels in static state, PNM/DOX and PNM/ LM/DOX were soaked in PBS at $37{ }^{\circ} \mathrm{C}$. As shown in Fig. 4B, hydrogels with or without LM droplets could release DOX slowly and steadily. These hydrogels can be well used as drug sustained release carrier. Furthermore, due to the excellent NIR laser responsive properties of PNM/LM/DOX, the release of DOX
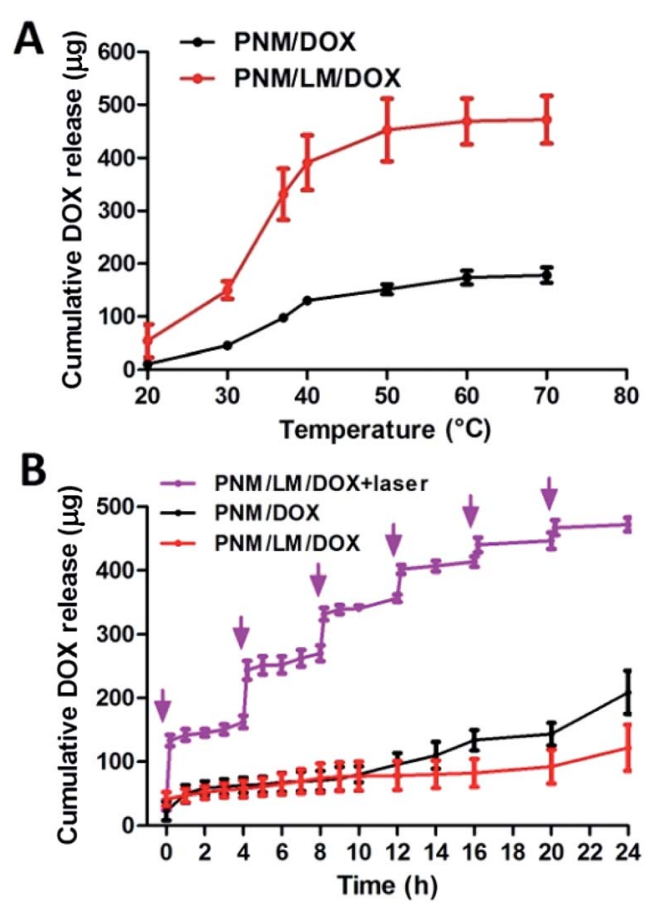

Fig. 4 DOX release from hydrogels. (A) In vitro DOX release profiles of PNM/DOX and PNM/LM/DOX in PBS at different temperature. (B) Cumulative DOX release $(\mu \mathrm{g})$ from PNM/DOX and PNM/LM/DOX in PBS $\left(\mathrm{pH} 7.4,37^{\circ} \mathrm{C}\right)$, and PNM/LM/DOX in response to NIR laser irradiation (arrows indicated the time points when NIR laser irradiation was executed for $12 \mathrm{~min}, 808 \mathrm{~nm}, 1.0 \mathrm{~W} \mathrm{~cm}^{-2}$ ). 
from hydrogels also could be readily accelerated via NIR laser irradiation, as indicated by the dramatic increase DOX release upon NIR laser irradiation at 0, 4, 8, 12, 16 and $20 \mathrm{~h}$ (NIR laser exposure every four hours, $808 \mathrm{~nm}, 1.0 \mathrm{~W} \mathrm{~cm}^{-2}$ ). With the NIR laser irradiation, LM droplets embedded in hydrogels networks could be heated up because of the photothermal conversion ability. When the temperature increased above LCST of hydrogels, they shrunk, squeezing out water and entrapped DOX at the same time. The amount of DOX released from hydrogels increased significantly when they were exposed to NIR laser for only $12 \mathrm{~min}$, which was much higher than that released without irradiation for $24 \mathrm{~h}$. Therefore, the PNM/LM/DOX has good photothermal conversion efficiency and could complete DOX release under NIR laser irradiation. Further, the sustained release of DOX from hydrogels can be achieved without NIR laser irradiation, and the controlled and rapid release of DOX from hydrogels can be achieved with NIR laser irradiation. So, the PNM/LM is an ideal carrier material for controlled drug release.

\section{In vitro cytotoxicity test of chemophotothermal therapy}

The chemophotothermal cytotoxicity of the PNM/LM/DOX against 4T1 cancer cells was detected by CCK-8 assay and live/ dead assay. As shown in Fig. 5A, there is no obvious difference between cells viabilities irradiated with or without NIR laser for $12 \mathrm{~min}$ (control and laser group). And cell viabilities of PNM and PNM/LM groups display no obvious decrease, indicating that NIR laser only is ineffective for 4T1 cancer cells killing and PNM/LM have no obvious cytotoxicity toward these cells. However, DOX-loaded PNM/LM hydrogels group has some cytotoxicity because there is a slow release of DOX from PNM/ LM/DOX. In addition, there is no obvious decrease of cell viabilities in NIR laser irradiated PNM hydrogels group (PNM + laser group), because PNM hydrogels have no photothermal conversion ability. But when PNM contained with LM droplets, they could raise the temperature to kill cancer cells under NIR laser irradiation. The cell viabilities in NIR laser irradiated group (PNM/LM + laser group) are much lower than cells in group without irradiation (PNM/LM group). The chemophotothermal synergistic therapy group (PNM/LM/DOX + laser group) exhibited a significant therapeutic effect compared with those single therapeutic groups. This group could raise temperature under NIR laser irradiation and promote DOX release to kill cancer cells simultaneously.

The therapeutic effect of photothermal and chemophotothermal groups is also shown in Fig. 5B, respectively, following exposure to the NIR laser irradiation $(808 \mathrm{~nm}, 1.0 \mathrm{~W}$ $\mathrm{cm}^{-2}$ ) for $0,1,2,5,8$ and $12 \mathrm{~min}$. From the results of photothermal group, they can demonstrate that the PNM/LM could respond to the NIR laser irradiation immediately. And increased temperature of hydrogels could contribute to kill cancer cells. Furthermore, the results of PNM/LM/DOX + laser group illustrate the superiority of chemophotothermal therapy powerfully. The PNM/LM/DOX can convert NIR laser irradiation into thermal energy and raise temperature up rapidly. The increase of temperature can kill cancer cells directly, and it also promote
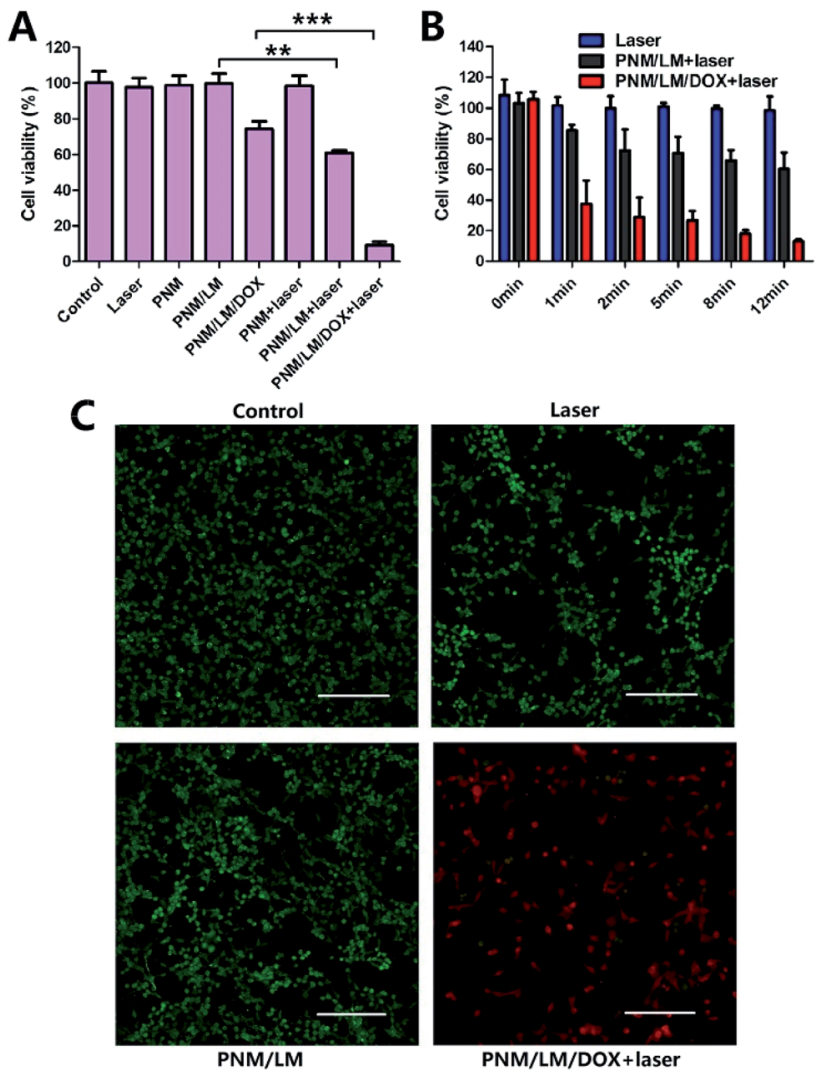

Fig. 5 In vitro cytotoxicity of hydrogels. (A) The cell viability of 4T1 cancer cells incubated with the PNM, PNM/LM and PNM/LM/DOX with or without NIR laser irradiation $\left(808 \mathrm{~nm}, 1.0 \mathrm{~W} \mathrm{~cm}^{-2}, 12 \mathrm{~min}\right)$. (B) The cell viability of $4 \mathrm{~T} 1$ cancer cells incubated with the PNM/LM and PNM/ LM/DOX exposed to NIR laser irradiation ( $\left.808 \mathrm{~nm}, 1.0 \mathrm{~W} \mathrm{~cm}^{-2}\right)$ for 0,1 , 2, 5, 8 and $12 \mathrm{~min}$. Data presented as means $\pm \mathrm{SD}(n=3, * * P<0.01$, $* * * P<0.001)$. (C) Live/dead cells image, scale bar: $200 \mu \mathrm{m}$.

to hydrogels shrinkage and DOX release. Therefore, the cell viabilities decreased to $37 \%$ at $1 \mathrm{~min}$ and $9 \%$ at $12 \mathrm{~min}$ dramatically. As shown in Fig. 5C, the result of live/dead assay also confirmed the excellent effect of chemophotothermal therapy.

\section{Conclusions}

In this study, we have developed a liquid metal-based poly(NIPAm-co-MBA) polymeric hydrogels for chemophotothermal synergistic cancer therapy. The hydrogel owns excellent NIR laser-stimulated and temperature responsive properties. Because of its porous 3D network structure and swelling properties, it can be adopted as a drug carrier and release DOX slowly and steadily. When the temperature is higher than LCST, the hydrogel can shrink rapidly and it would also squeeze DOX out synchronously. The introduction of LM droplets can facilitate the rapid formation of hydrogels and improve the photothermal conversion efficiency. The release of DOX from the PNM/LM/ DOX could be readily accelerated via NIR laser irradiation. Therefore, the sustained release of DOX from hydrogels can be achieved without NIR laser irradiation, and the controlled and 
rapid release can be achieved with NIR laser irradiation. The in vitro cytotoxicity test demonstrated the good biocompatibility of PNM/LM. Although there are no obvious signs of toxicity for these hydrogels, one needs to further evaluate the toxicity of hydrogels formation in order to facilitate subsequent in vivo applications. Furthermore, the addition of LM can also be used in imaging diagnosis and treatment, and the good drug loading ability of this hydrogel can make it encapsulate more kinds of drugs not only limited by chemotherapeutic drugs. Furthermore, the drug-loaded PNM/LM carriers can be placed in a designated position to achieve targeted drug release under the guidance of NIR laser irradiation, with the aim of reducing damage to surrounding normal tissues. So, this NIR laser and temperature dual responsive hydrogel is an ideal carrier for targeted and controlled drug delivery systems.

\section{Conflicts of interest}

There are no conflicts to declare.

\section{Acknowledgements}

This work was financially supported by the National Natural Science Foundation of China Key Project under Grant No. 91748206 and 51890893.

\section{Notes and references}

1 K. Pal, A. K. Banthia and D. K. Majumdar, Des. Monomers Polym., 2009, 12, 197-220.

2 J. J. Grodzinski, Polym. Adv. Technol., 2010, 21, 27-47.

3 F. Zhao, D. Yao, R. Guo, L. Deng, A. Dong and J. Zhang, Nanomaterials, 2015, 5, 2054-2130.

4 D. Seliktar, Science, 2012, 336, 1124-1128.

5 T. Thambi, V. H. G. Phan and D. S. Lee, Macromol. Rapid Commun., 2016, 37, 1881-1896.

6 J. F. Mano, Adv. Eng. Mater., 2008, 10, 515-527.

7 C.-L. Lo, K.-M. Lin and G.-H. Hsiue, J. Controlled Release, 2005, 104, 477-488.

8 Z. Xiao, Y. Tan, J. Ma, D. Wang, L. Yu, F. Yue, S. Xu and J. Huang, J. Appl. Polym. Sci., 2018, 46375.
9 Y. Zhang, J. Ji, H. Li, N. Du, S. Song and W. Hou, Soft Matter, 2018, 14, 1789-1798.

10 F. Oroojalian, M. Babaei, S. M. Taghdisi, K. Abnous, M. Ramezani and M. Alibolandi, J. Controlled Release, 2018, 288, 45-61.

11 X. Xu, Z. Huang, Z. Huang, X. Zhang, S. He, X. Sun, Y. Shen, M. Yan and C. Zhao, ACS Appl. Mater. Interfaces, 2017, 9, 20361-20375.

12 A. M. Gobin, E. M. Watkins, E. Quevedo, V. L. Colvin and J. L. West, Small, 2010, 6, 745-752.

13 M. Sun, J. Guo, H. Hao, T. Tong, K. Wang and W. Gao, Theranostics, 2018, 8, 2634-2645.

14 D.-K. Lim, A. Barhoumi, R. G. Wylie, G. Reznor, R. S. Langer and D. S. Kohane, Nano Lett., 2013, 13, 4075-4079.

15 M. Hashemi, M. Omidi, B. Muralidharan, H. Smyth, M. A. Mohagheghi, J. Mohammadi and T. E. Milner, ACS Appl. Mater. Interfaces, 2017, 9, 32607-32620.

16 S. A. Chechetka, Y. Yu, X. Zhen, M. Pramanik, K. Pu and E. Miyako, Nat. Commun., 2017, 8, 15432.

17 L. Yi and J. Liu, Int. Mater. Rev., 2017, 62, 415-440.

18 J. Yan, Y. Lu, G. Chen, M. Yang and Z. Gu, Chem. Soc. Rev., 2018, 47, 2518-2533.

19 X. Wang and J. Liu, Micromachines, 2016, 7, 206-209.

20 X. Wang, Y. Zhang, R. Guo, H. Wang, B. Yuan and J. Liu, J. Micromech. Microeng., 2018, 28, 034003.

21 Y. Yu and E. Miyako, Chem.-Eur. J., 2018, 24, 9456-9462.

22 X. Sun, B. Yuan, W. Rao and J. Liu, Biomaterials, 2017, 146, 156-167.

23 S. H. Jeong, K. Hjort and Z. Wu, Sensors, 2014, 14, 1631116321.

24 Y. Lu, Q. Hu, Y. Lin, D. B. Pacardo, C. Wang, W. Sun, F. S. Ligler, M. D. Dickey and Z. Gu, Nat. Commun., 2015, 6, 10066.

25 X. Li, M. Li, L. Zong, X. Wu, J. You, P. Du and C. Li, Adv. Funct. Mater., 2018, 28, 1804197.

26 X. Sun, M. Sun, M. Liu, B. Yuan, W. Gao, W. Rao and J. Liu, Nanoscale, 2019, 11, 2655-2667.

27 N. Taccardi, M. Grabau, J. Debuschewitz, M. Distaso, M. Brandl, R. Hock, F. Maier, C. Papp, J. Erhard, C. Neiss, W. Peukert, A. Görling, H.-P. Steinrück and P. Wasserscheid, Nat. Chem., 2017, 9, 862-867. 